\title{
EL ÚLTIMO PROYECTO DE FOUCAULT: UNA REHABILITACIÓN DE LA AMISTAD
}

Francisco Guerrero Ortega*

SINTESE - Para Foucault, a amizade val além da divisão tradicional entre eros e filía, tornando possível o estabelecimento de uma forma de vida a partir de uma escolha sexual. Os homossexuais em particular possuem a oportunidade histórica de utilizar sua sexualidade para a constituição de novas formas de existência. Os heterossexuais deveriam experimentar um "devir homossexual" que também conduzisse à procura de formas alternativas de sociedade.

\begin{abstract}
For Foucault, friendship goes beyond the traditional division between eros and philia, making it possible to design a lifestyle departing from a sexual decision. Homosexuals in particular have a historic chance to use their sexuality in order to construct new ways of being. Heterosexuals should experiment with "becoming homosexual", which also leads to the search for alternative forms of society.
\end{abstract}

Después de haber estudiado la historia de la sexualidad creo que se debería intentar comprender la historia de la amistad, o de las amistades (Foucault 1985c, 55).

\section{Actualización de la estilística de la existencia}

La investigación genealógica foucauldiana de la antigua estética de la existencia no constituye un mero análisis histórico, adquiere más bien el carácter de un programa ético-político. Establecer un equilibrio entre estos dos elementos va a provocar, como voy a intentar demostrar, una cierta tensión en el texto.

Los escritos de Foucault tienen por objetivo la rehabilitación de la noción de filosofía qua Askesis propia de la antigüedad, como se pone de manifiesto sobre todo en la introducción del Uso de los placeres. Este proyecto se integra según Foucault en el ámbito de los trabajos históricos a pesar de no ser realizados con los ojos de un historiador. Se trata del "protocolo de un ejercicio", del ejercicio de la propia transformación. La actualización de la estilística de la existencia no significa la incorporación del modelo ético greco-romano. La antigüedad representa

- Universidade Federal de Goiás, UFGO.

\begin{tabular}{|l|l|l|l|l|l|}
\hline VERITAS & Porto Alegre & v. 42 & $n^{2} 1$ & Março 1997 & p. 105-116 \\
\hline
\end{tabular}


para él "un error profundo"; los griegos no son ni admirables (1984c, 38) ni ofrecen una alternativa: "La solución de un problema no se encuentra en la solución de otro problema que fue planteado por otra gente en otra época" (1987a, 268). Foucault no busca un principio olvidado que la filosofia debe redescubrir (1985a, 22), resalta sin embargo la importancia del contacto con la filosofia antigua y subraya el valor ejemplar de semejantes experiencias éticas (1987a, 271).

¿Cómo se puede explicar la reticencia a adoptar el modelo ético de la antigüedad poniéndolo al mismo tiempo como ejemplo? La razón radica en el hecho de que Foucault, a pesar de contemplar una posible actualización de la estética de la existencia, pretende situarla en el marco sobreindividual de la amistad.

La antigüedad presenta una experiencia moral centrada en una relación consigo mismo (ética) no relatada a ningún sistema social, jurídico o institucional. La situación actual muestra un paisaje similar: "La mayoría de nosotros ya no cree que la ética se fundamente en la religión, pero tampoco queremos que un sistema jurídico intervenga en nuestra vida moral, personal, privada. Los movimientos de liberación actuales sufren por el hecho de no poder encontrar un fundamento sobre el que se pudiese basar la elaboración de una nueva ética" (1987a, 267).

El problema de una ética entendida como la forma que se le puede dar a la propia vida gana actualidad (1984d, 21). La idea del bios como materia de una obra de arte fascina a Foucault. ${ }^{1}$ El sujeto se constituye mediante "prácticas analizables históricamente" existentes en toda cultura (1987a, 273, 289; 1985a, 19). Como consecuencia concibe Foucault el cuidado de sí como "punto de resistencia preferente y útil contra el poder político" y localiza el objetivo político en el fomento de nuevas formas de subjetividad. El individuo alcanza autonomía mediante las prácticas del sí y mediante la unión de la propia transformación con los cambios sociales y políticos. Esta relación consigo mismo autónoma y no normativizada no debe ser entendida en un sentido liberal, ${ }^{3}$ constituye más bien una subjetividad anárquica (Schürmann 1986, 305-8), pues de lo que se trata en último término es de liberarse del estado y de las formas de subjetivación ligadas al estado (como va a poner de manifiesto el análisis de la amistad).

Foucault aboga por la creación de nuevos esbozos de sí mismo que no tengan obligatóriamente como resultado la formación de un sujeto dúctil: "Se podría imaginar la constitución de una existencia que no sea especialmente buena en el uso

Foucault 1987a, 272ss.; 1988b, 14. Incluso el suicidio va a ser contemplado desde esta perspectiva (1984a, 56ss.). Wilhelm Schmid ha investigado en varias publicaciones la anulación de la división entre arte y vida (1988, 348ss.; 1991a, 650; 1991b, 82-87; 1991c, 243, 302ss.; 1993a, 74ss.).

3 1985b, 54. Una afirmación que va a ser relativizada dos años después, lo que no lleva consigo la pérdida de importancia del cuidado de sí, sino más bien la introducción de la diferenciación entre relaciones de poder y estados de dominio (1985a, 27).

3 Richard Rorty (1992, 328-33) siente que la recepción francesa interprete a Foucault como "anarquista" y no como un "buen liberal", como él y W.E. Connolly hacen (1993, 151-58) - este último mediante la comparación de la "sensibilidad foucauldiana" con un "ethos democrático." En este contexto resulta acertada la siguiente observación de James Miller $(1993,384)$ : "El trabajo de Foucault durante toda su vida, tal y como yo lo he llegado a comprender, es mucho más inconvencional - y mucho más disconforme - que lo que están dispuestos a admitir algunos de sus admiradores "progresivos." A no ser que esté profundamente equivocado Foucault representó un desafio básico y. audaz frente a todo lo que parece ser correcto en la cultura occidental - incluyendo casi todo lo que parece ser correcto entre un gran número de académicos americanos de izquierdas." 
corriente de la palabra, pero que sea muy intensiva" (Schmid/Schmidt 1993b, 132). Este es un hecho que hay que tomar en consideración. ${ }^{4}$ Foucault era un "guerrero" (Veyne 1986, 934) y la utilidad de la filosofía reside en "hacer la guerra" (Ibid., 937). La "guerra" como metáfora de la sociedad, acuñada por Foucault en los años setenta, sigue teniendo validez en los ochenta (Honneth/Seitter 1989, 24ss.).

La amistad es la forma de existencia contemplada por Foucault cuando piensa en una posible actualización de la estética de la existencia (1984c, 41); a pesar de limitar el análisis de la misma casi únicamente a la cultura homosexual, hablando así de un "estilo de vida gay" (1988d, 292, 301ss.; 1985c, 54) - lo que por otra parte no excluye una ampliación a otros grupos. Se trata de llegar a una nueva forma de existencia por medio de la sexualidad (1984e, 85-90; 1984b, 109ss.). Esta forma de existencia alcanzable mediante un cierto trabajo sobre sí, una cierta askesis (1984b, 110; 1984e, 88) toma forma en la amistad (1984e, 85ss., 89; 1985c, 54ss.).

\section{Amistad y estilística de la existencia}

La ocupación con la problemática de la amistad goza de una buena coyuntura en la actualidad. Foucault no tuvo tiempo de realizar un análisis arqueológicogenealógico de este fenómeno, a pesar de haber realzado su importancia (1985c, 55). Subraya, de manera análoga a ciertos estudios sociológicos contemporáneos, las diferentes nociones de la amistad en el transcurso histórico. Una historia de la amistad pondría de manifiesto - como fue el caso de la historia de la subjetividad - las diferentes formas adoptadas por este fenómeno. La amistad representa hoy en día una posibilidad de utilizar el espacio abierto por la pérdida de vínculos orgánicos, de experimentar con la multiplicidad de posibles formas de vida. Para ello no ofrece Foucault ninguna receta, sin embargo concentra su atención sobre diferentes formas de existencia: cultura homosexual, vanguardias artísticas, etc.

En los dos últimos libros publicados por Foucault no se hace referencia a la amistad, a pesar de que ésta gozase de gran importancia en la antigüedad. En las últimas entrevistas, por el contrario, va a ser mencionada en el marco de una posible rehabilitación de la estética de la existencia. ¿Se trata de un nuevo anacronismo? Mi tesis al respecto es la siguiente: la noción de amistad constitutiva para la antigüedad se encontraba ante el dilema de poder introducir la reciprocidad en la relación amical únicamente mediante la supresión de las relaciones sexuales. La antigua ética sexual, caracterizada por actividad, asimetria y obligación de penetración, no ofrece ningún lugar para la philia - especialmente desde que Platón concibiese la reciprocidad sólo a expensas del elemento de eros. La separación de philia y eros permanece constante en toda la historia de la amistad. Precisamente

4 En la antigüedad constituía la estética de la existencia un asunto de muy pocos; estaba limitada a un pequeño grupo que pretendía donar a su vida de una forma bella. Cuando se piensa en su posible actualización debe plantearse la cuestión del elitismo y los peligros potenciales que lleva consigo semejante rehabilitación, como ponen de manifiesto los intentos de poner al día la estilística de la existencia por el dandismo o el anarquismo politico. Deleuze (1991a, 165; 1993a, 135) da en el centro de la cuestión caracterizando al sujeto como "modo de la intensidad." 
esta historia pretende Foucault invertir, pues su rehabilitación del fenómeno de la amistad como forma de la estilística de la existencia intenta recuperar el elemento de eros, rechazado desde la antigüedad, para la dinámica de la amistad.

Foucault permanece en la entrevista con Dreyfus y Rabinow en una noción tradicional de la amistad. La razón por la cual no se tematiza la amistad en la Historia de la sexualidad no estriba, como Dreyfus y Rabinow creen $(1987,305)$, en el hecho de que Foucault no preste atención a las prácticas marginales en su insistencia sobre los sistemas: "No se olviden de que el Uso de los placeres es un libro sobre ética sexual y no un libro sobre amor, amistad o reciprocidad. [...] La amistad es mutua, las relaciones sexuales no lo son. [...] Cuando se tiene una amistad es difícil tener relaciones sexuales" $(1987 a, 269)$. O sea, el esquema de la dualidad tradicional eros contra philia. Sin embargo, dice Foucault en la misma entrevista: "Mi pregunta es la siguiente: ¿Podemos darnos una ética de las acciones y de sus placeres susceptible de considerar el placer del otro? ¿Es el placer del otro algo que puede ser integrado en nuestro propio placer - sin relación a la ley, al matrimonio y a no se que más?" (Ibid., 270). En estas lineas se pasa de una descripción histórica de las relaciones amicales en la antigüedad greco-latina a su posible actialización como una relación afectiva intersubjetiva capaz de respetar el placer del otro. De esto precisamente trata la diferente "economia de los cuerpos y sus placeres" qua resistencia frente al poder moderno añorada en las últimas páginas de La voluntad de saber. ${ }^{5}$

La amistad practicada en la antigüedad carece de interés para Foucault por dos razones:

a. Se trataba por una parte de un tipo de relación institucionalizada, el cual no dejaba espacio para la experimentación: "Las relaciones amicales [...] jugaron un papel importante, sin embargo había para ellas un marco institucional suplementario - con un sistema de coerciones, tareas, obligaciones mutuas, una jerarquía entre amigos, etc. No creo que debamos imitar semejante modelo" (1982b, 38).

La amistad tenía en la pólis clásica griega una fución de integración social. Relaciones de amistad constituían los átomos de la pólis y la condición de su supervivencia: "Sea cual sea la importancia de los valores emocionales y expresivos para la amistad, no constituyen ningún grupo en sí sino que pertenecen al sistema de las relaciones sociales que la regulan y gobiernan por fuera (mediante leyes) y por dentro (mediante el ideal de virtud)". ${ }^{6}$ Hoy en día va a ser factible, mediante procesos de liberación y de diferenciación, "arreglárselas sin el amor por lo orgánico", ya que existen "posibilidades de escaparse de los vínculos orgánicos de la

- El mérito de Dreyfus y Rabinow consiste en haber realzado la amistad como elemento de resistencia en el marco de una nueva "economia de los cuerpos y los placeres." Sin embargo parecen haber descuidado dos aspectos importantes: por una parte el hecho de que Foucault ya habia pensado en la amistad como "fundamento para una descripción efectiva de una forma no reactiva de resistencia"; por otra que Foucault somete al fenómeno de la amistad a un proceso de transformación que permite recuperar el componente de eros.

- Kon (1979, 40, 44). Cfr. Onfray 1993, 236; Nötzoldt-Linden 1994, 37ss.; Easterling 1989, 11ss.; Fraisse 1974, 202ss. En la actualidad sostiene el interaccionismo simbólico de Suttles (1979) una posición similar tratando la amistad como "institución social." Para Foucault, por el contrario, ofrece la amistad una posibilidad de trazar "lineas de fuga" (Deleuze) en el edificio institucional. 
religión, la familia, el trabajo y la comunidad que antigüamente garantizaban la coexión de muchas sociedades" (Sennett 1984, 31). El fenómeno de la amistad va a ser atractivo sobre todo en un contexto moderno: "Donde la sociedad más bien tradicional establece las relaciones afectivas normativamente y las tareas de la amistad están institucionalizadas, permite la sociedad individualizada la producción autónoma de relaciones intimas con las personas más diferentes y en los más distintos ámbitos de interés" (Nötzoldt-Linden 1994, 216).

b. La amistad en la antigüedad se basaba en la omisión de las relaciones sexuales. Rehabilitarla significa para Foucault introducir el componente de eros en la relación de amistad, lo que por otra parte no significa que toda amistad tenga un carácter sexual. Sin embargo deben ser tomados en consideración los siguientes puntos: 1. La discusión de la amistad tiene lugar en el marco del análisis de nuevas formas de vida homosexual, pues "la homosexualidad ofrece la ocasión histórica de reabrir las posibilidades existentes de relaciones y sentimientos, lo cual no sucede como consecuencia de las cualidades "verdaderas" de los homosexuales, sino porque ésta se encuentra en una posición "transversal" permitiendo el trazado de diagonales en el tejido social, que permitan la aparición de estas posibilidades" (1984e, 90). 2. Las prácticas sadomasoquistas (como componente importante de una ascesis homosexual) ofrecen a Foucault un modelo especial para su noción de amistad (presencia y control de relaciones de poder, reciprocidad, fijación situativa de reglas, etc.). 3. La rehabilitación foucauldiana de la amistad se basa en el hecho de "utilizar la sexualidad para la creación de múltiples relaciones" (Ibid., 86). 4. Una nueva "economia de los cuerpos y los placeres" debe superar la división existente desde la antigüedad entre deseo y placer y el consiguiente privilegio del deseo, en beneficio de una relación que apunte a la intensificación mútua del placer. Por lo tanto se equivoca Wilhelm Schmid (1991c, 346) al afirmar que "la ética de la amistad en la que piensa Foucault y que tiene el carácter de una ética de la simpatía no puede ser definida mediante relaciones sexuales." El mismo error comete Frederic-Yves Jeannet $(1994,33)$ cuando pretende analizar la noción foucauldiana de amistad criticando al mismo tiempo el libro de James Miller, The passion of Michel Foucault, debido a la relación establecida entre sadomasoquismo y filosofia. La única definición de amistad propuesta por Foucault (en el contexto de la discusión de formas de existencia homosexuales) es la siguiente: "Amistad, es decir, la suma de todas las cosas mediante las cuales se puede obtener un placer mútuo" (1984e, 87).

Foucault subraya la ambivalencia entre amistad y amor en el cristianismo. La amistad tenía un carácter sospechoso y el amor era la forma de liberarse de ella (Althusser 1993, 309ss.). El primado de la amistad como virtud suprema en la antigüedad desaparece con la propagación del cristianismo. Especialmente Agustín representa el arquitecto de esta transformación. En el intenso vínculo humano representado por la amistad se reconoce un elemento de egoismo, contemplado como un desvio del amor a Dios (Nötzoldt-Linden 1994, 39; McNamara 1964, 106). Agustín se plantea el problema de reconciliar la philia con el amor a Dios. La philia de la antigüedad va a ser substituida por el agape y el amor al prójimo (el prójimo es un individuo abstracto): "El amor al prójimo es más una actitud moral que una 
forma de relación. El desvio de todo lo terrenal, corpóreo, interhumano y el traslado de la amistad al interior del individuo y a su actitud espiritual lleva consigo una pasividad social, a pesar de ser esta amistad relativamente intelectual. Mediante oración y sacrificio para los amigos apenas se pone en peligro el sistema establecido, mediante solidaria acción cotidiana, por el contrario, si" (Nötzoldt-Linden 1994, 48).

Otros comentarios de Foucault sobre la historia de la amistad se limitan a la descomposición de las sociedades monosexuales en el siglo XVIII (1984b, 99). La desaparición de la amistad se remite al hecho de que ésta desempeñaba ciertas tareas socio-políticas en la sociedad aristocrática, interrumpidas con la implantación de nuevas estructuras políticas: "El ejercito, la burocracia, la administración, la enseñanza (en el sentido moderno de la palabra) no pueden funcionar con relaciones intensas. Creo que se puede observar en todas estas instituciones un cierto esfuerzo por reducir o limitar estas relaciones afectivas" (1985c, 55).

Mientras la amistad fue aceptada social y culturalmente no representaba la homosexualidad ningún problema; pero desde que la amistad se desmorona como forma de relación tolerada culturalmente gana actualidad la siguiente pregunta: “¿Qué hacen los hombres juntos?” La homosexualidad se convierte en un problema médico y socio-político. Estas consideraciones (para Foucault únicamente de carácter hipotético) son de gran relevancia al plantear la cuestión acerca de la forma de existencia implicada en toda decisión sexual.

\section{Hacia una ascesis y una forma de vida homosexual}

La amistad tiene para Foucault principalmente el sentido de una amistad homosexual. Su atención se concentra sobre todo en las posibilidades de las que dispone la cultura homosexual hoy en día para crear nuevas formas de amistad. La democratización de la estilística de la existencia, como Hans Herbert Kögler (1994, 190) propone, no puede realizarse en la amistad ya que su potencial transgresivo reside precisamente en su naturaleza minoritaria. Democratizarla supondría otorgarla un carácter mayoritario (Deleuze 1994, 205).

Los análisis foucauldianos de la antigua estilística de la existencia le conducen a la rehabilitación y revaloración de la antigua praxis ascética. La askesis es la tarea de la autoelaboración. En la discusión actual sobre la amistad debe jugar la askesis un papel importante, ya que mediante las prácticas del si ${ }^{7}$ se puede llegar a una askesis homosexual, la cual "nos permita trabajar sobre nosotros mismos e inventar (no digo descubrir) un modo de vida que todavía nos parezca improbable" $(1984$ e, 88). Las decisiones sexuales tienen una dimensión existencial, atraviesan la totalidad de la vida y son susceptibles de transformarla. Se trata por lo tanto de crear formas de existencia a través de estas decisiones: "Diría símplemente que se

\footnotetext{
El concepto de práctica del sí ofrece una denominación diferente y actualizada de la askesis, la cual incluye en la cultura homosexual prácticas sadomasoquistas (como un juego de poder recíproco que aspira a un placer desexualizado más allá del discurso de la sexualidad). Cfr. Foucault 1985c, 55; 1988d, 298ss.; Kögler 1994, 149ss.; Miller 1993, 253ss.; Champagne 1991, 185ss. He preferido la utilización del término griego original askesis el cual tiene un sentido más general y positivo que el de ascesis, generalmente utilizado en un contexto cristiano.
} 
debe emplear la sexualidad para descubrir y crear nuevos tipos de relaciones. Ser homosexual significa ser en devenir" (1984b, 109-10). Por lo tanto se convierte cađa vez más el problema de la homosexualidad en un problema de la amistad (1984e, 86).

Foucault compara las relaciones heterosexuales institucionalizadas con el carácter abierto de las relaciones homosexuales, en las cuales se encuentran los hombres "unos frente a otros sin armas ni palabras adecuadas, sin algo que pueda confirmar el sentido del movimiento que les atrae mútuamente. Deben inventar una relación desde la $\mathrm{A}$ a la $\mathrm{Z}$ que todavía carece de forma: la amistad" (Ibd., 87. Itálica: FGO). Los conceptos de forma de vida (homosexual) y de amistad permiten rechazar toda una cultura homosexual concentrada sobre la liberación del deseo y la búsqueda de la propia identidad sexual, en favor del uso de la homosexualidad para la creación de nuevas formas de existencia: "El concepto de forma de vida me parece importante. ¿No se debería introducir una diferenciación más subtil que no procediese según clases sociales, grupos profesionales o niveles culturales, sino que se orientase hacia el tipo de relación, o sea, hacia el "modo de vida"? Un modo de vida puede ser compartido por individuos que se diferencian en relación a la edad, al status y a la actividad social. Puede conducir a relaciones intensivas que no se igualan a ninguna relación institucionalizada. Y un modo de vida puede culminar, creo yo, en una ética y una cultura" (1984e, 89). La ética de la amistad apunta a intensificar la experimentación (1988e, 165). La experimentación como fundamento ético se concentra en la percepción y aumento del placer propio y el del otro (el del amigo), en vez de en una hermenéutica del deseo. Foucault encuentra este fenómeno en la cultura homosexual de Nueva York y San Francisco, en ciudades donde han surgido "laboratorios de experimentación sexual" (1988d, 298).

\section{Función de la amistad en la ética negativa de Foucault}

El viraje ético realizado por Foucault en los años ochenta es al mismo tiempo estético con el objetivo de realzar la posible creación de relaciones sociales, ya que no existe nada dado o natural en nuestra pertenencia a determinados grupos sociales. Si la reflexión sobre el fenómeno de la amistad supone, como yo pienso, el punto final de la meditación ético-política foucauldiana (la cual apunta hacia la actualización de la estilística de la existencia) ésta puede realzar únicamente la - capacidad de formación estética de las relaciones humanas.

La ética de la amistad sólo puede ser una ética negativa cuyo programa debe ser vacio, es decir, capaz de ofrecer "herramientas para relaciones multiformes, variables y concebidas individualmente" (1984e, 92). Cada individuo debe formar su propia ética; la ética de la amistad prepara el camino para la creación de formas de vida, sin prescribir un modo de existencia como el correcto. Proponer un programa $^{8}$ significaría prohibir otros esbozos y sobre todo relativizar la formación

B La idea del programa "vacio" (1984e, 92ss.) es de central importancia en la ética foucauldiana. Proponer un programa lleva consigo la normativización de determinados modos de existencia. Interesante resulta la noción de "programa vacio" la cual conserva la forma, la idea (en este caso la 
individual: "No quisiera desanimar la experimentación, no me gustaría que los homosexuales dejaran de creer que la organización de sus propias relaciones depende de ellos mismos mediante el descubrimiento de lo que es adecuado en su situación" (1988d, 301-2). El hecho de que Foucault hable sobre formas concretas de amistad y dirija su amistad hacia determinadas formas de vida, no quiere decir que estos modos de existencia sean los correctos. Si se subrayan ciertas formas de vida sucede, únicamente, porque precisamente estos modos de existencia expresan la noción de "programa vacio", como sucede por ejemplo en 1968.

Para Foucault representa la amistad una relación con el otro que no tiene la forma ni de unanimidad consensual ni de violencia directa. Es una relación agonística opuesta a un antagonismo esencial, una "relación que es al mismo tiempo incitación mútua y lucha, tratándose no tanto de una oposición frente a frente como de una provocación continua" (1982c). Relaciones agonísticas son relaciones libres que apuntan al desafio y a la incitación recíproca y no al sometimiento del otro.

El poder es un juego estratégico. La nueva ética de la amistad procura jugar dentro de las relaciones de poder con un mínimo de dominación y crear un tipo de relación intenso y móbil que no permita que las relaciones de poder se conviertan en estados de dominación. Precisamente este juego con el poder (como posibilidad de dirigir y transformar el comportamiento del otro) hace de la amistad algo fascinante: "Cuanto más libre sea la gente en su relación con los otros más grande serán las ganas de determinar el comportamiento del otro. Cuanto más abierto sea el juego más seductor y fascinante será" (1985a, 27-8). Una noción de amistad, como la foucauldiana, atravesada por relaciones de poder contradice la idea común en la sociología y en la filosofía social, según la cual la amistad representa una relación voluntaria basada en la transparencia y la verdad de la información (Nötzoldt-Linden 1994, 149). Sin embargo desigualdad, jerarquía y rúpturas son componentes importantes de la amistad.

Michel Onfray $(1993,233)$ ha definido adecuadamente la amistad como electiva, aristocrática y asocial subrayando así su carácter desigual y jerárquico. La amistad definida de esta manera se opone a los principios democráticos que han conducido a su codificación. La amistad se encuentra por encima del derecho, de las leyes, de la familia y de las instancias sociales: "Se es amigo antes de ser ciudadano y a veces a pesar del status de ciudadania. De ahí deviene su función radical atómica y su carácter asocial" (Ibid., 235).

\section{Amistad como forma libre de sociedad}

Las posibilidades creativas de la homosexualidad no se agotan si se contempla su lucha como una lucha por la igualdad de derechos con los heterosexuales (matrimonio, adopción, herencia, etc.). ${ }^{9}$ Esto supondría la ampliación a círculos

creación posible de nuevos tipos de relaciones), creando una "cavidad" que puede ser rellenada en cada caso, según el individuo, de forma diferente.

2 Foucault (1988d, 288ss.) menciona que el intento de los homosexuales de organizarse como una clase social según el modelo político ha fracasado, porque los homosexuales no constituyen ninguna clase social. 
homosexuales de las formas de sociedad reducidas en la actualidad a los heterosexuales, en vez de inventar nuevas formas de existencia no institucionalizables: "Qué se le permita a alguien hacer lo que quiera en nombre del respeto por los derechos individuales me parece estupendo. Pero si lo que queremos es la creación de una nueva forma de vida entonces es irrelevante la cuestión de los derechos individuales. De hecho vivimos en un mundo legal, social e institucional en el que las únicas relaciones posibles son extremamente pocas, extremamente simplificadas y extremamente pobres. Naturalmente existen las relaciones fundamentales del matrimonio y la familia, pero ¿cuantas relaciones podrían existir si fuesemos capaces de encontrar sus propias leyes no en las instituciones sino en otros portadores? lo que por supuesto no sucede" (1982b, 38).

Según Foucault vivimos en un mundo en el que las instituciones sociales han contribuido a limitar el número posible de relaciones. La razón de esta restricción reside en el hecho de que una sociedad que permitiese el crecimiento de las relaciones posibles sería más dificil de administrar y de controlar. La lucha homosexual debe (aquí reside su potencial transgresivo ampliable a otros tipos de conflictos sociales: movimientos antiracistas o feministas, etc.) aspirar a la creación de un nuevo "derecho relacional", que permita todo tipo posible de relaciones en vez de impedirlas o bloquearlas.

La posibilidad de constituir formas nuevas de sociedad es también posible para la comunidad heterosexual, la cual tiene que ser inciuida en la lucha por un nuevo "derecho relacional" (Ibid., 39). Debido a su carácter "minoritario" los homosexuales están capacitados para efectuar un devenir creativo que permita la construcción de nuevas formas de relación, un "devenir homosexual" que afecte incluso a los heterosexuales (Deleuze 1993b, 246ss.; 1994, 205ss.; Deleuze/Guattari 1980); pues precisamente reside una importante posibilidad de resistencia en el "devenir homosexual" del heterosexual, esto es, en el dejarse afectar por su potencial. Este carácter minoritario se opone diametralmente a la demanda de igualdad de derechos característica de la mayor parte de los grupos homosexuales (así como de los feministas y antiracistas). La dimensión político-transgresiva de la amistad reside en el rechazo de las formas impuestas de relación y de subjetividad. Ya en 1977 subraya Foucault los logros del movimiento feminista, el cual ha sido capaz de distanciarse del discurso del "aparato de la sexualidad" y de orientarse hacia nuevas formas de comunidad y de placer. En la discusión feminista (Friedmann 1989, 275ss.) tiene el concepto de amistad un significado importante ofreciendo una alternativa al discurso siempre retornante de la liberación sexual. La amistad representa una posibilidad de constituir la comunidad y la sociedad al nivel individual de un tipo de relación libre y no institucionalizado basada en la reciprocidad y en la confianza.

El proyecto foucauldiano de una ética de la amistad en el contexto de una posible actualización de la estética de la existencia permite transcender el marco de la autoelaboración individual para colocarla en una dimensión colectiva. La amistad supera la tensión existente entre el individuo y la sociedad mediante la creación de un espacio intersticial (una subjetivación de grupo) susceptible de considerar tanto necesidades individuales como objetivos colectivos y de subrayar 
su interacción. Procesos de subjetivación dan cuenta de la producción de formas de vida y de sociedad, así como de la creación de nuevas posibilidades de existencia (Deleuze 1987, 145; 1991a, 143; 1991b, 155ss.; 1993c, 166; Deleuze/Parnet 1980, 100). No todas las subjetivaciones tienen un tipo de sujeto por objetivo, existen subjetivaciones sin sujeto (de tipo acontecimiento) y subjetivaciones colectivas a las que pertenece la amistad. El proyecto de una ética de la amistad consiste en la búsqueda de lugares de producción de subjetividad: "¿Cua es nuestra ética? ¿Cómo producimos una existencia artística? ¿Cuales son nuestros procesos de subjetivación no reducibles a nuestros códigos morales? [...] ¿Se puede esperar algo de las comunidades actuales?" (Deleuze 1993c, 165). En torno a estas preguntas debe girar la reflexión sobre la amistad en la actualidad.

Resumiendo se puede decir que los intentos de actualización de la antigua estilística de la existencia culminan en una ética de la amistad. La amistad supera para Foucault la división tradicional eros/philia y conlleva la posibilidad de constituir una forma de vida a partir de una decisión sexual. Especialmente los homosexuales poseen la oportunidad histórica de utilizar su sexualidad para crear nuevas formas de comunidad. Estos nuevos tipos de relación y de sociedad multiformes y comprendidos bajo la noción de amistad se oponen a las formas de relación prescritas y normativizadas. Por lo tanto el objetivo de la lucha homosexual (también de la feminista y antiracista) no debe consistir en la exigencia de igualdad de derechos, sino en la cración de un nuevo derecho relacional. Los heterosexuales deben experimentar un "devenir homosexual" que también conduzca a la búsqueda de formas alternativas de sociedad.

Lamentablemente la recepción foucauldiana ha ignorado hasta ahora la problemática de la amistad. Realzar este importante elemento de su filosofía supone la ocasión de comprender la dimensión filosófico-social del pensamiento de Foucault. Un pensamiento que no culmina en el individualismo, como muchos afirman, sino que intenta introducir movimiento y fantasía en las deterioradas y rígidas relaciones sociales. Foucauit pretende mostrar con su rehabilitación de la amistad que las posibles formas de vida en común en nuestra sociedad no se agotan en la familia o en el matrimonio. Es posible crear nuevas formas de existencia productoras de una intensidad y un placer especial. Aquí reside el desafio de una reflexión sobre la amistad y la herencia de Michel Foucault.

\section{Bibliografía}

ALTHUSSER, L., 1993: Die Zukunft hat Zeit. Frankfurt/Main.

CHAMPAGNE, J., 1991: "Interrupted Pleasure: A Foucauldian Reading of Hard Core / A Hard Core (Mis)Reading of Foucault". En: Boundary 18 (2).

CONNOLLY, W. E., 1993: The Augustinian Imperative. Newbury Park/Ca.

DELEUZE, G., 1987: Foucault. Frankfurt/Main.

- 1991a: "Das Leben als Kunstwerk". En: SCHMID, W. (Ed.): Denken und Existenz bei Michel Foucault. Frankfurt/Main.

, 1991b: "Was ist ein Dispositiv?". En: EWALD, F., y WALDENFELS. W. (ed.): Spiele der Wahrheit. Michel Foucaults Denken. Frankfurt/Main.

, 1993a: "Die Dinge aufbrechen, die Worte aufbrechen". En: Unterhandlungen 1972/1990. Frankfurt/Main. 
- 1993b: “Kontrolle und Werden". En: Unterhandlungen 1972/1990. Frankfurt/Main.

- , 1993c: "Ein portraet Foucaults". En: Unterhandlungen 1972/1990. Frankfurt/Main.

_ - 1994: "Philosophie und Minoritaet". En: VOGL, J. (Ed.): Gemeinschaften. Positionen zu einer Philosophie des Politischen. Frankfurt/Main.

DELEUZE, G. y Guattari, F., 1992: Tausend Plateaus. Berlin.

DELEUZE, G. y PARNET, C., 1980: Dialoge. Frankfurt/Main.

DREYFUS, H. y RABINOW. P., 1987: Jenseits von Strukturalismus und Hermeneutik. Frankfurt/Main.

EASTERLING, P., 1989: "Friendship and the Greeks". En: PORTER, R. y TOMASELLI (Ed.): The Dialectics of Friendships. London.

FOUCAULt, M., 1980: “The Confession of the Flesh". En: Power/Knowledge. New York.

- , 1982a: "Conversation avec Wemer Schroeter". En: Courant, G.: Wemer Schroeter. Paris.

- , 1982b: "The Social Triumph of Sexual Will". En: Christopher Street, 64.

- 1984a: "Ein ganz harmloses Vergnuegen". En: Von der Freundschaft. Berlin.

- , 1984b: "Geschichte und Homosexualitaet". En: Von der Freundscchaft. Berlin.

- , 1984c: "Le retour de la morale". En: Les nouvelles littéraires, 28.6.

— , 1984d: "Le souci de la vérité". En: Magazine littéraire, 207.

- , 1984e: "Von der Freundschaft als Lebensweise”. En: Von der Freundschaft. Berlin.

—_ 1985a: "Freiheit und Selbstsorge". En: Freiheit und Selbstsorge. Frankfurt/Main.

- , 1985b: "Hermeneutik des Subjekts". En: Freiheit und Selbstsorge. Frankfurt/Main.

, 1985c: "Que fabriquent donc les hommes ensemble?". En: Le Nouvel Observateur, 22.11.

- , 1987a: "Zur Genealogie der Ethik". Epílogo a Dreyfus, H. y Rabinow, P.: Jenseits von Strukturalismus und Hermeneutik. Frankfurt/Main.

- , 1987b: "Jenseits von Gut und Boese. Gespraech mit den Studenten". En: Von der Subversion des Wissens. Frankfurt/Main.

- , 1987c: "Das Subjekt und die Macht". Epilogo a Dreyfus, H. y Rabinow, P.: Jenseits von Strukturalismus und Hermeneutik. Frankfurt/Main.

- 1988a: "Le gay savoir". En: MEC magazine 6-7.

- 1988b: "The Minimalist Self". En: Politics, Philosophy, Culture 1977-1984. New York, London.

don.

, 1988c: "Practicing Criticism". En: Politics, Philosophy, Culture 1977-1984. New York, Lon-

, 1988d: "Sexual Choice, Sexual Act: Foucault and Homosexuality". En: Politics, Philosophy, Culture 1977-1984. New York, London.

— , 1988e: "Social Security". En: Politics, Philsophy, Culture 1977-1984. New York, London.

FOUCAULt, M. y Sennett, R., 1984: „Sexualitaet und Einsamkeit”. En: Von der Freundschaft. Berlin.

FRAISSE, J.-C., 1974: Philia. La notion d'amitié dans la philosophie antique. Paris.

FRIEDMAN, M., 1989: "Feminism and modem Friendship: Dislocating the Community". En: Ethics 99.

HONNETH, A. y SEITTER, W., 1989: „Foucault = Kant minus X?”. En: Spuren 26/27.

JEANNEt, F.-Y., 1994: "La muerte de Michel Foucault". En: La Jomada Semanal 250, 27.3.

KOEGLER, H. H., 1994: Michel Foucault. Stuttgart. Weimar.

KON, I. S., 1979: Freundschaft. Geschichte und Sozialpsychologie der Freundschaft als soziale Institution und als individuelle Beziehung. Hamburg.

McNAMARA, M. A., 1964: Friends and Friendship for Saint Augustine. New York.

MILLEr, J., 1993: The Passion of Michel Foucault. New York.

NOETZOLDT-LINDEN, U., 1994: Freundschaft. Zur Thematisienung einer vernachlaessigten soziologischen Kategorie. Opladen.

ONFRAY, M., 1993: La sculpture de soi. La morale esthétique. Paris.

RORTY, R., 1992: "Moral identity and private autonomy". En: Michel Foucault Philosopher. New York, London.

SCHMID, W., 1988: “Exerzitien der Existenz. Kunst und Lebenskunst bei Edgar Degas". En: SCHMID, W. (Ed.): Wege zu Edgar Degas. Muenchen.

- 1991a: "Das Dasein - ein Kunstwerk. Zum Verhaeltnis von Kunst und Lebenskunst bei Nietzsche". En: Deutsche Zeitschrift fuer Philosophie 6.

- 1991b: "Kunst und Leben. Anmerkungen zu einer wieder auflebenden Diskussion". En: Merkur 502. 
1991c: Auf der Suche nach einer neuen Lebenskunst. Frankfurt/Main.

1993a: Was geht uns Deutschland an? Frankfurt/Main.

SCHMID, W. y SCHMIDT, H., 1993b: "Philsophieren ueber Lebenskunst". En: Deutsche Zeitschrift fuer Philosophie 1.

SCHUERMANN, R., 1986: "On constituting oneself an anarchistic subject". En: Praxis International 6. SUTTLES, G. D., 1970: "Friendship as a social institution". En: McCall, G.J. y otros (Ed.): Social Relationships, Chicago.

VEYNE, P., 1986: "Le dernier Foucault et sa morale". En: Critique 471/472.I 